\title{
Outbreak of Shiga toxin-producing Escherichia coli O157:H7 linked to raw drinking milk resolved by rapid application of advanced pathogen characterisation methods, England, August to October 2017
}

Juli Treacy ${ }^{1}$, Claire Jenkins², Karthik Paranthaman³, Frieda Jorgensen4, Doris Mueller-Doblies ${ }^{5}$, Muna Anjum5, Lukeki Kaindama6, Hassan Hartman ${ }^{1}$, Miranda Kirchner ${ }^{5}$, Therese Carson ${ }^{5}$, Ishani Kar-Purkayastha ${ }^{1}$

1. Public Health England South East, Hampshire and Isle of Wight Health Protection Team, Fareham, United Kingdom

2. National Infection Service, Public Health England, London, United Kingdom

3. National Infection Service, Field Epidemiology Service, Public Health England, London, United Kingdom

4. National Infection Service, Food Water and Environmental Microbiology Laboratory, Public Health England, Porton, United Kingdom

5. Animal and Plant Health Agency, Surrey, United Kingdom

6. Gastrointestinal Emerging and Zoonoses Infections, Public Health England, London, United Kingdom

Correspondence: Ishani Kar-Purkayastha (ishani.karpurkayastha@phe.gov.uk)

Citation style for this article: Treacy Juli, Jenkins Claire, Paranthaman Karthik, Jorgensen Frieda, Mueller-Doblies Doris, Anjum Muna, Kaindama Lukeki, Hartman Hassan, Kirchner Miranda,
Carson Therese, Kar-Purkayastha Ishani. Outbreak of Shiga toxin-producing Escherichia coli O157:H7 linked to raw drinking milk resolved by rapid application of advanced pathogen characterisation methods, England, August to October 2017. Euro Surveill. 2019;24(16):pii=1800191. https://doi.org/10.2807/1560-7917.

ES.2019.24.16.1800191

An outbreak of Shiga toxin-producing Escherichia coli (STEC) $0157: \mathrm{H}_{7}$ occurred on the Isle of Wight between August and October 2017. Of the seven cases linked to the outbreak, five were identified through the statutory notification process and two were identified through national surveillance of whole genome sequencing data. Enhanced surveillance questionnaires established a common link to a farm, and link to the likely food vehicle, raw drinking milk (RDM). Microbiological investigations, including PCR, identified the presence of STEC $0157: \mathrm{H}_{7}$ in samples of RDM. Analysis of core genome single nucleotide polymorphism (SNP) data of STEC 0157:H7 from human stool specimens, animal faecal samples and RDM demonstrated a one SNP difference between isolates, and therefore close genetic relatedness. Control measures that were put in place included suspension of sales and recall of RDM, as well as restrictions on public access to parts of the farm. Successful integration of traditional epidemiological surveillance and advanced laboratory methods for the detection and characterisation of STEC $0_{157}: \mathrm{H}_{7}$ from human, animal and environmental samples enabled prompt identification of the outbreak vehicle and provided evidence to support the outbreak control team's decision-making, leading to implementation of effective control measures in a timely manner.

\section{Background}

Shiga toxin-producing Escherichia coli (STEC) belong to a group of zoonotic pathogens transmitted to humans by direct contact with contaminated food, animals or their environments, or by secondary person-to-person spread, particularly in family groups within households [1]. The natural reservoir of STEC is the gastrointestinal tract of ruminant animals; in the United Kingdom (UK), the predominant animal reservoirs are cattle and sheep. STEC cause a wide spectrum of illness ranging from mild to severe bloody diarrhoea. Haemolytic uraemic syndrome (HUS), a complication of STEC, develops in $5-15 \%$ of cases, depending on the age and sex of the case [2].

STEC are defined by the presence of the Shiga toxinencoding genes stx 1 and $s t \times 2$, which can be divided into subtypes stx1a-1d and stx2a-2g [3]. STEC can be classified into serotypes, with STEC $0157: \mathrm{H}_{7}$ being the commonest STEC serotype associated with human disease in England, with 600-800 cases reported annually [1].

STEC 0157:H7 emerged as a pathogen of public health concern in the 1980s, when it was found to be the cause of HUS outbreaks in children [4]. To counter this threat to public health, epidemiological and microbiological surveillance systems were put in place in the UK in the 1990 , and continue to be developed and enhanced [5]. In 2009, the National Enhanced STEC Surveillance System (NESSS) was implemented at Public Health England (PHE) to collect epidemiological data on every case of STEC 0157: $\mathrm{H7}$ in England and Wales [1]. Isolates of STEC 0157:H7 detected at local diagnostic 


\section{FIGURE 1}

Confirmed and probable cases in STEC O157:H7 outbreak by week of symptom onset, England, $2017(n=7)$

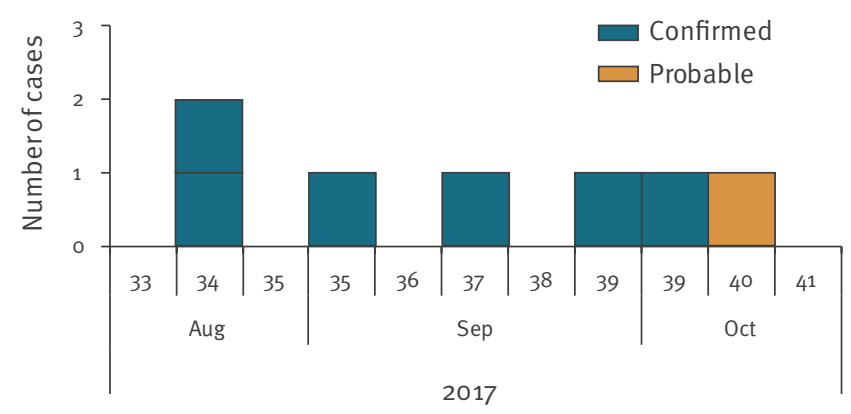

Week of symptom onset

STEC: Shiga toxin-producing Escherichia coli.

laboratories are referred to the Gastrointestinal Bacteria Reference Unit (GBRU) at PHE for confirmatory testing and typing. Since 2015, whole genome sequencing (WGS) has been employed on all STEC submitted to GBRU to provide highly discriminatory typing for public health surveillance and to facilitate outbreak detection and investigation [6].

Food-borne outbreaks of STEC $0157: \mathrm{H}_{7}$ are difficult to detect and investigate, as they often involve a small number of geographically dispersed cases [7]. Studies have shown that the use of WGS for routine surveillance of STEC $0157: \mathrm{H}_{7}$ is a robust approach for the identification of geographically and temporally distinct cases sharing common exposures [6].

\section{Outbreak detection}

In the outbreak described here, the first two cases-who were both residents of the Isle of Wight, off the south coast of England-were notified to PHE by local microbiologists on detection of presumptive E. coli $\mathrm{O}_{157}: \mathrm{H}_{7}$ in stool samples. A common epidemiological link between the cases and a local farm was established on 25 September 2017, and an outbreak control team (OCT) meeting convened later the same day. Just before the OCT meeting, a further case with an isolate genetically identical to the first notified case was identified through routine review of the PHE WGS database.

This report summarises the key findings, actions and conclusions of the multi-faceted outbreak investigation, and aims to highlight the role of advanced laboratory tools such as PCR and WGS in informing a public health response.

\section{Methods}

Case ascertainment by enhanced epidemiological surveillance

Presumptive cases of STEC were reported directly to PHE centres by clinical microbiologists at local hospital laboratories and a standardised STEC Enhanced Surveillance Questionnaire (STEC ESQ) (https://www. gov.uk/government/uploads/system/uploads/attachment_data/file/323423/VTEC_Questionnaire.pdf) was administered to each case either by local health protection professionals or environmental health officers (EHOS). Data from the questionnaires were uploaded to NESSS. NESSS was reviewed to identify any cases with an epidemiological link to the Isle of Wight that had been notified nationally since the beginning of January 2017. Any cases identified this way, or as having a microbiological link through STEC WGS surveillance processes, were reviewed against the outbreak case definitions.

For the purposes of the outbreak investigation, a confirmed case was defined either as (i) a case with STEC O157:H7 PT21/28 with a single nucleotide polymorphism (SNP) profile within 5 SNPs of the outbreak strain profile and reported onset of symptoms since August 2017 or (ii) a case with HUS and serum antibodies to the lipopolysaccharide of $E$. coli $\mathrm{O}_{157}$ or a faecal specimen that tested positive for the stx gene by PCR, notified since August 2017 with a known epidemiological link to the implicated farm. Known epidemiological links included consumption of raw drinking milk (RDM) produced at the farm or being an employee or close contact of an employee at the farm. A probable case was defined as a case with HUS with a known epidemiological link to the farm, in the absence of a positive STEC microbiological result.

Microbiological examination of food samples Samples of RDM from the implicated farm-three from the bulk tank and six from packaged bottles-were taken by EHOs on 25 September 2017. Samples of pasteurised whole, semi-skimmed and skimmed milk from the holding tanks and from bottles were also obtained from the farm by EHOs. All samples were collected and transported in accordance with the Food Standards Agency Food Law Code of Practice (https://www.food. gov.uk/enforcement/codes-of-practice/food-law-codeof-practice-2015). They were transported to the PHE Food, Water and Environmental (FWE) Microbiology Laboratory, Porton, in cold boxes (temperature $0-8^{\circ} \mathrm{C}$ ) and tested within 24 hours of collection.

Tests for the detection of STEC (including STEC 015 $7: \mathrm{H}_{7}$ ), Salmonella species, Campylobacter species and Listeria species were performed on $25 \mathrm{~mL}$ samples of milk. Enumeration of coliform bacteria, E. coli, coagulase-positive staphylococci, aerobic colony count and Listeria species (including L. monocytogenes) was carried out using dilutions of milk samples [8]. Real-time PCR was used to examine samples for the 
presence of STEC 0157 based on CEN/ISO TS 13136, as described previously [9]. Enrichment broths that were PCR positive for stx were sub-cultured onto MacConkey agar and cefixime tellurite sorbitol MacConkey agar, and up to 50 colonies were retested using the same PCR assay.

\section{Veterinary investigation and microbiological examination of animal faecal specimens}

A Veterinary Investigation Officer (VIO) from the Animal and Plant Health Agency (APHA) visited the implicated farm on 5 October 2017 to assess the potential role of the farm's animals as the source of infection, and to review if there were appropriate controls in place to reduce the risk of contamination of RDM from the farm environment. Faecal samples were collected from calves and cows, and were tested using immuno-magnetic separation culture methodology, as described by Pritchard et al. [10].

\section{Dairy hygiene inspection}

The Food Standards Agency (FSA) Dairy Hygiene Inspectorate (DHI) carried out independent environmental inspections at the farm, focusing on raw milk production activity. This included collection of further raw milk samples to establish whether there was ongoing contamination of the RDM. These samples were also sent to the PHE FWE laboratory for testing.

\section{Molecular typing of STEC O157:H7 by whole genome sequencing}

Isolates of STEC 0157: $\mathrm{H}_{7}$ from clinical specimens, food and animal samples were sent to PHE GBRU for confirmation, phage typing and WGS $[6,11]$. DNA was extracted from cultures for sequencing on the HiSeq 2500 instrument (Illumina, California, USA). High quality Illumina reads were mapped to the STEC 0157: $\mathrm{H} 7$ reference genome Sakai (GenBank accession BAooooo7) using BWA-MEM [12]. SNPs were identified using GATK2 [13] in unified genotyper mode. Core genome positions that had a high quality SNP (>90\% consensus, minimum depth $10 \times, G Q \geq 30$ ) in at least one isolate were extracted. SNP positions that were present in at least $80 \%$ of isolates were used to derive maximum likelihood phylogenies with RaxML [14] using the GTRCAT model with 1,000 iterations.

Genomes were compared with the sequences held in the PHE STEC 0157: $\mathrm{H}_{7}$ WGS database. This database comprises genomes from more than 2,500 cultures of STEC 0157:H7 submitted to GBRU between1982-2017. Hierarchical single linkage clustering was performed on the pairwise SNP difference between all isolates at various distance thresholds $(\Delta 250, \Delta 100, \Delta 50, \Delta 25$, $\Delta 10, \Delta 5, \Delta 0)$. The result of the clustering is a SNP address that can be used to describe the population structure based on clonal groups. Clusters at the zero, five and 10 SNP level are highlighted for further investigation and are analysed in the context of their nearest neighbours. Isolates of STEC $0157: \mathrm{H}_{7}$ with less than five SNPs differences within their core genome were considered closely related and likely to have an epidemiological link [6]. stx subtyping was performed, as described by Ashton et al. [15].

FASTQ reads from all sequences in this study and the PHE STEC 0157:H7 WGS data can be found at the PHE Pathogens BioProject at the National Center for Biotechnology Information (Accession PRJNA248792).

\section{Ethical statement}

The planning, conduct and reporting of this study was in line with the Declaration of Helsinki, as revised in 2013.

\section{Results}

\section{Descriptive epidemiology}

A total of seven cases (six confirmed and one probable) were identified as part of this outbreak, with onset of symptoms between August-October 2017 (Figure 1). STEC 0157:H7 was isolated from the six confirmed cases. The probable case had HUS and drank RDM from the implicated farm, but did not provide any specimens for microbiological tests. Four of the cases were male and three were female. The median age was 10 years (range: 1-62 years; mean: 21 years) (Figure 2). The duration of symptoms ranged from 2-17 days (mean: 7 days; median: 6 days).

Five of the seven cases were residents of the Isle of Wight and were notified to PHE's Hampshire and Isle of Wight Health Protection Team by local clinicians in line with statutory requirements either on detection of presumptive $E$. coli $\mathrm{O} 157$ in stool samples at the local hospital or on presentation with HUS. There were two cases resident in a different part of the country; these were both initially identified as likely to be linked to the outbreak through routine review of PHE'S STEC O157 WGS database, which showed isolates from these cases to be genetically identical to the isolate from case 1 (i.e. zero SNP difference between them) (Figure 3). Sequences from other closely related isolates were more than 10 SNPs different from the outbreak strain sequence and were not temporarily related or geographically or epidemiologically linked to the implicated farm.

Review of STEC ESQs confirmed that all seven cases were either residents of, or had visited, the Isle of Wight within the 7 days before symptom onset. Four cases provided a clear history of consuming RDM from the farm. For a further two cases, there was no clear recollection of whether the cases themselves had consumed the milk. However, both had close relatives who consumed RDM from the farm. One case did not report any epidemiological link to the farm or RDM produced at the farm.

All three cases of HUS had symptom onset after the recall advice was communicated to customers. One case reported that they might have consumed RDM 


\section{FIGURE 2}

Confirmed and probable cases in STEC O157:H7 outbreak by age and sex, England, $2017(\mathrm{n}=7)^{\mathrm{a}}$

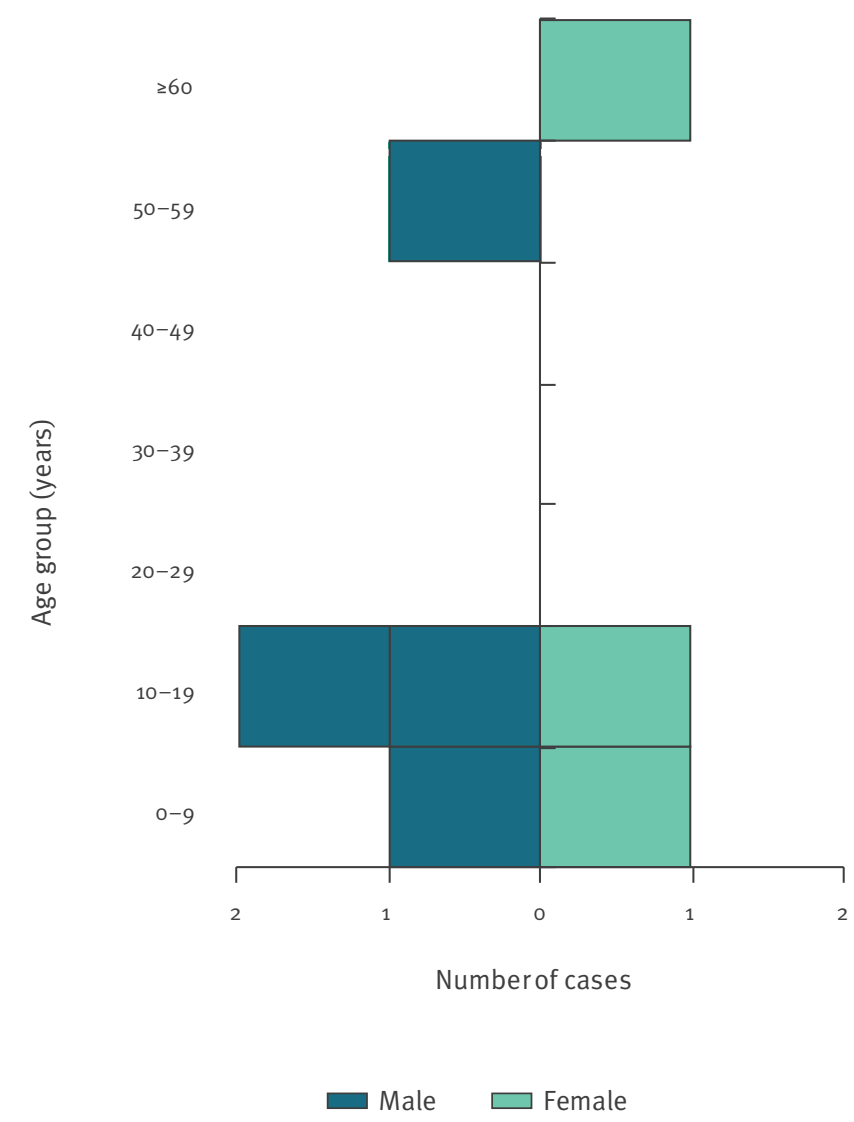

STEC: Shiga toxin-producing Escherichia coli.

${ }^{a}$ One probable case.

after the recall advice was issued. Another case was unsure if they consumed RDM, but they belonged to a household where RDM was consumed by other family members. The third case reported consuming RDM despite being aware of the recall advice.

\section{Environmental investigations}

The farm was primarily a dairy farm producing raw and pasteurised milk, cheese and butter. The site included a café, a petting area, an educational centre, holiday accommodation and a shop selling produce from the farm. Overall, the standard of cleanliness at the farm was assessed as very good. It was noted that there was a dedicated car park for visitors with direct access to the café and farm shop. The visitors' car park and all concrete pathways around the café and farm shop were dry and clean, and no signs of faecal contamination were seen anywhere near these areas. There was no livestock on neighbouring fields and there were no footpaths across the farm land. There was therefore no contact between grazing cattle from the farm and any other livestock, nor potential for the general public to come in contact with grazing cattle. Animal accommodation was assessed as being in a very good state of repair, with spacious and well-ventilated pens for calves and heifers, as well as plenty of bedding. Following the inspection, the installation of further hand washing facilities in the petting area was recommended.

Independent $\mathrm{DHI}$ inspections at the farm, with a focus on raw milk production, did not identify any hygiene concerns.

\section{Microbiological investigations of food and animals samples \\ STEC 0157:H7 PT21/28 was isolated from six of the} seven cases linked to this outbreak. Colonies of STEC O157:H7 PT21/28 were detected by PCR from each of the three bulk tank milk samples taken on 25 September 2017. Four subsequent RDM samples from the bulk tank, taken on three separate occasions at least a week apart, were negative. Of note, statutory indicator bacteria tests gave compliant results for all three samples from which STEC 0157: $\mathrm{H}_{7}$ was isolated. The standard for RDM is plate count at $30^{\circ} \mathrm{C}(\mathrm{cfu} / \mathrm{mL})<20,000$ and coliforms $(\mathrm{cfu} / \mathrm{mL})<100$.

No other pathogens were detected in any of the milk samples tested.

Of the animal faecal samples, 13 calf and eight cow samples tested positive for STEC $0157: \mathrm{H}_{7}$. Analysis of the WGS data confirmed that all isolates from the clinical specimens, food and animal samples were different by one SNP (Figure 3).

\section{Outbreak control measures}

The farm management team agreed to voluntarily cease sale of the RDM on 25 September 2017, and to contact customers who had purchased RDM from the farm advising them not to consume the product and to return it. This was in line with the OCT's advice, along with the recommendation for suspension of animal petting activities until the end of the investigation. Occupational health measures were also put in place to prevent cross-contamination from the farm animals to the food business operations, and to minimise direct and indirect exposure of staff and their families to animal faeces.

Controls on the sale of RDM were maintained throughout the course of the investigation, during which time the $\mathrm{DHI}$ issued a formal letter to the farm owner instructing the withdrawal of the raw milk from sale. The FSA Incidents branch provided support to the DHI and coordinated the FSA response. Given the high prevalence of the outbreak strain in animal faecal samples and the likely resources involved in mitigating the risks, the farm decided to voluntarily relinquish their authorisation to sell RDM. 


\section{FIGURE 3}

Phylogeny of the clinical, food and farm isolates linked to the STEC O157:H7 outbreak, England, 2017

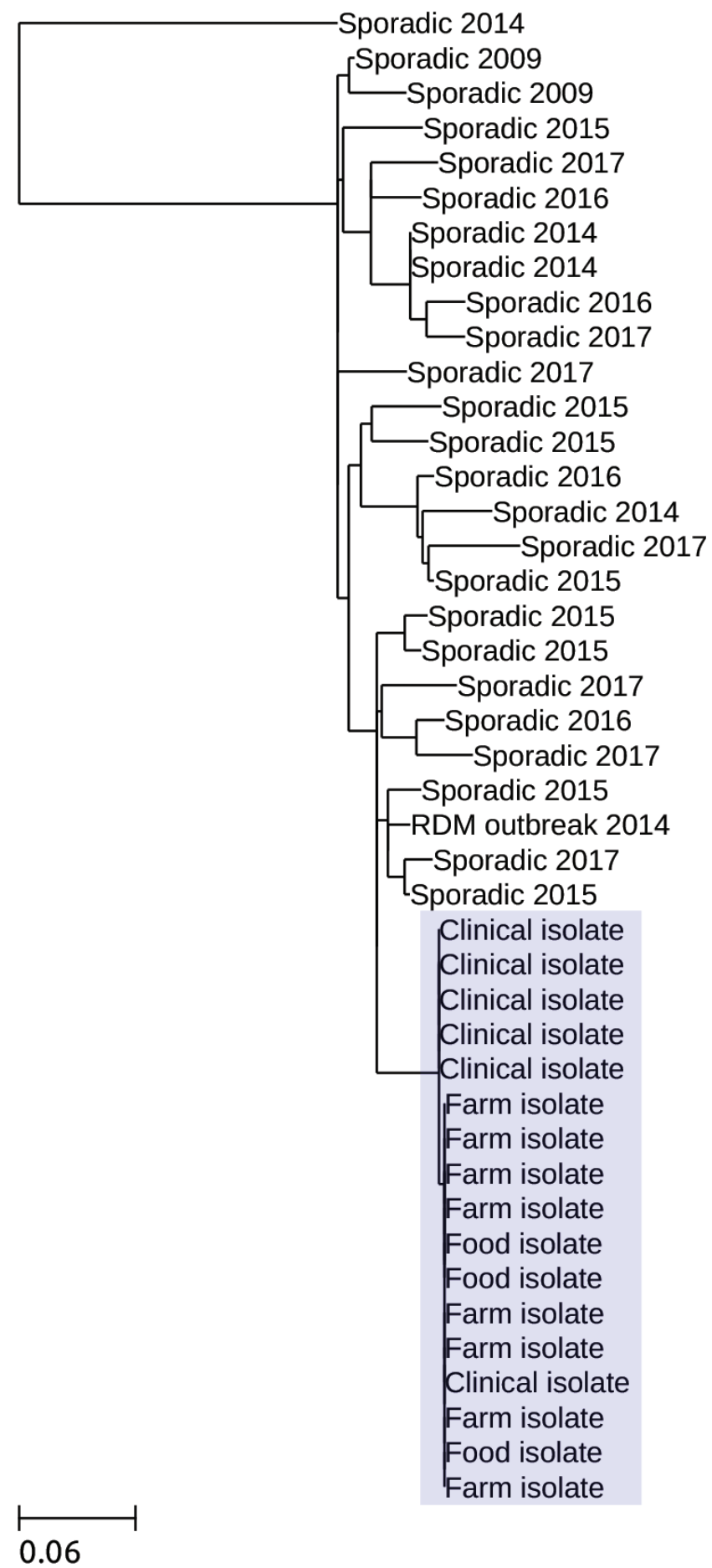

STEC: Shiga toxin-producing Escherichia coli.

Isolates linked to the outbreak are highlighted. The clinical isolates were from the six confirmed cases. Sequences from other closely related sporadic isolates (sporadic isolates defined here as not linked to the outbreak) within the 25 single nucleotide polymorphism (SNP) single linkage cluster available in the Gastrointestinal Bacteria Reference Unit archive are shown for context, including an isolate from an outbreak associated with raw drinking milk in 2014 in the south-west of England. Quality trimmed Illumina reads were mapped to the STEC $0_{157}$ reference genome Sakai (Genbank accession number: BA000007) using BWA-MEM. SNPs were identified using GATK2 in unified genotyper mode. Core genome positions that had a high quality SNP $(>00 \%$ consensus, minimum SNP ( $90 \%$ cons were extracted. GTRCAT model with 1,000 iterations.

\section{Discussion}

During the outbreak, the rapid detection by PCR of STEC O157: $\mathrm{H}_{7}$ from the RDM samples provided the OCT with robust evidence that RDM was the vehicle of infection, and supported the OCT's decisions on control measures put in place on the sale and recall of RDM. The enhanced sensitivity of the PCR approach compared to culture alone, in combination with timely sampling of the RDM at the farm, contributed to the successful isolation of the outbreak strain in the contaminated product. The timely sampling of the RDM was initiated following the identification of the epidemiological link early in the course of the investigation.

STEC is notoriously difficult to detect and isolate from food samples, as the infectious dose is low and contamination levels are often below the detection limit of the tests [7]. In recent years, PCR has been used successfully to detect STEC $\mathrm{O}_{157: \mathrm{H}_{7}}$ in food samples during outbreaks caused by contaminated watercress [9], cross-contamination at two butchers' premises [16] and contaminated frozen beef burgers (data not shown), as well as in surveillance studies on RDM [17] and imported curry and banana leaves [18].

Due to restrictions on the sale of RDM that only allow direct sales from the farm to the final consumer (and not via an intermediate retailer), milk-borne outbreaks associated with RDM in the UK are smaller than those caused by pasteurisation failures and, therefore, are more difficult to detect [19]. Although the first two local cases in this outbreak were detected by analysis of the enhanced surveillance data, two further casesboth of whom were resident in a different region of the country-were identified by routine surveillance using WGS data. Consumption of RDM was not reported by all cases; however, in most instances there was evidence that RDM from the farm was available in the home environment and was consumed by members of the household. This highlights a common problem encountered during outbreak investigations, where interviewees fail to recall an accurate food history or may be unaware they consumed the implicated product [7].

A strength of this investigation was the use of STEC WGS surveillance systems to identify and assess cases in England within five SNPs from the designated outbreak profile. WGS is a highly discriminatory typing method that can be used for case ascertainment, regardless of the confounding influence of inaccurate food histories $[16,19]$.

Previous studies have shown that different Stx subtypes are associated with varying clinical outcomes, with Stx2a most likely to be associated with severe disease [20]. Although PCR was available for identification of the different Stx subtypes before the implementation of WGS, the assay was labour intensive and the results were difficult to interpret; as a result, the PCR assays were not used routinely at PHE. Since the implementation of WGS, the Stx subtype has been routinely 
available as part of the downstream bioinformatics pipeline [15]. The strain associated with this outbreak had stx2a, and the OCT was informed at the beginning of the investigation that it was highly likely to cause severe disease, including HUS. This information contributed to the risk management of the outbreak, providing further evidence that suspension of the sale of RDM and animal petting activities, as well as the product recall, were justified. The timely product recall advice undoubtedly prevented further cases.

In England, Wales and Northern Ireland, farms may sell RDM directly to the consumer on the farm, in a farmhouse catering operation, via door-to-door delivery service, through the Internet or at farmers' markets [21]. Restrictions on the sale of RDM are governed by the Food Hygiene (England) Regulations (2006) [22]. In a recent study of RDM carried out by PHE between 2014 and 2016, 454 per 770 (59.0\%) samples of RDM collected from retail sales in England for routine monitoring were of a satisfactory quality [17].

Between 2012 and 2017, the volume of RDM produced in the UK has increased fivefold [23]; during this period, there has also been an increase in gastrointestinal outbreaks associated with the consumption of unpasteurised dairy products, several having been reported since 2014 [23,24]. Prior to 2014, the last outbreaks associated with RDM in the UK occurred in 2002 [23].

In this outbreak, five of the seven cases were children. Severe symptoms of gastrointestinal disease caused by STEC 0157 are seen more frequently in younger children [1]. It is of concern that the families were not aware of the risk or, if they were, that they felt the risk was acceptable. Consumers of RDM may be influenced by information promoting the perceived benefits of RDM, without balancing this against the risk of foodborne infection [25]; this situation highlights the issue of risk perception more generally [26]. The FSA is currently working to improve the risk communication for RDM, including introducing revised labels with clearer warnings for vulnerable groups, including children and people with underlying health conditions [23].

The farm implicated in this outbreak was classed as small, with only cattle farmed. The farm had produced RDM for several decades, and no previous incidents had been recorded. During the outbreak investigation, no changes to the manufacturing protocols were identified. However, it was established that although the farm operation had always been 'closed', i.e. with no acquisition of livestock from other farms, 4 months before the outbreak a small number of animals were brought onto the farm from a neighbouring farm. Although they were accommodated in a separate area with reportedly no mixing, it is possible that there may have been indirect cross-contamination and that the outbreak strain was introduced into the indigenous herd from these visiting animals.

\section{Conclusions}

The outbreak investigation described here demonstrates how recent advances in laboratory technology, specifically PCR for the detection of STEC in food and WGS for case ascertainment and highly discriminatory strain typing, can inform outbreak detection and investigation. Successful integration of traditional epidemiological surveillance and advanced laboratory methods for characterisation of human, animal and environmental samples enabled identification of the outbreak vehicle, leading to implementation of effective control measures in a timely manner.

\section{Acknowledgements}

We would like to thank Francesco Tripodo, Dawn Hedges and Michael Wright at GBRU; Lisa Byrne, PHE national STEC surveillance lead; Drs. Rob Davies and Nick Duggett at Animal and Plant Health Agency for epidemiological and WGS advice. We would also like to acknowledge everyone who was part of the outbreak control team, including the Food Standards Agency and its Dairy Hygiene Inspectorate, and the IOW Council Environmental and Public Health teams.

This work was supported by the National Institute for Health Research Health Protection Research Unit in Gastrointestinal Infections and the Department for Environment, Food and Rural Affairs, Scottish Government and Welsh Government under APHA devolved surveillance contract B.

The views expressed are those of the author(s) and not necessarily those of the NHS, the NIHR, the Department of Health, Public Health England or the Department for Environment, Food and Rural Affairs.

\section{Conflict of interest}

None declared.

Authors' contributions

Juli Treacy, Claire Jenkins, Karthik Paranthaman and Ishani Kar-Purkayastha jointly wrote the manuscript. Juli Treacy and Ishani Kar-Purkayastha led the outbreak investigation. Claire Jenkins provided testing, analysis and interpretation of results on all human samples and WGS on isolates from human, food and animal samples. Karthik Paranthaman and Lukeki Kaindama were responsible for regional and national epidemiological support and surveillance, respectively. Frieda Jorgensen was responsible for the analysis of food samples and provided interpretation and commentary on the results of these samples. Doris Mueller-Doblies undertook the veterinary investigations on site and provided interpretation and commentary on findings. Muna Anjum, Miranda Kirchner and Therese Carson contributed to the testing and analysis of animal faecal samples including the application of WGS methods. Hassan Hartman developed the figure of the phylogenetic tree and graphical representation of relatedness of isolates. All authors contributed to the discussion and reviewed the manuscript. All authors read, commented upon and approved the final version of the paper.

\section{References}

1. Byrne L, Jenkins C, Launders N, Elson R, Adak GK. The epidemiology, microbiology and clinical impact of Shiga toxinproducing Escherichia coli in England, 2009-2012. Epidemiol 
Infect. 2015;143(16):3475-87. https://doi.org/10.1017/ So950268815000746 PMID: 25920912

2. Launders N, Byrne L, Jenkins C, Harker K, Charlett A, Adak GK. Disease severity of Shiga toxin-producing E. coli 0157 and factors influencing the development of typical haemolytic uraemic syndrome: a retrospective cohort study, 2009-2012. BMJ Open. 2016;6(1):eo09933. https://doi.org/10.1136/ bmjopen-2015-009933 PMID: 26826153

3. Scheutz F, Teel LD, Beutin L, Piérard D, Buvens G, Karch H, et al. Multicenter evaluation of a sequence-based protocol for subtyping Shiga toxins and standardizing Stx nomenclature. Clin Microbiol. 2012;50(9):2951-63. https://doi.org/10.1128/ JCM.00860-12 PMID: 22760050

4. Taylor CM, White RH, Winterborn MH, Rowe B. Haemolyticuraemic syndrome: clinical experience of an outbreak in the West Midlands. Br Med J (Clin Res Ed). 1986;292(6534):1513-6. https://doi.org/10.1136/bmj.292.6534.1513 PMID: 3087499

5. Adams NL, Byrne L, Smith GA, Elson R, Harris JP, Salmon R, et al. Shiga Toxin-Producing Escherichia coli 0157, England and Wales, 1983-2012. Emerg Infect Dis. 2016;22(4):590-7. https:// doi.org/10.3201/eid2204.151485 PMID: 26982243

6. Dallman TJ, Byrne L, Ashton PM, Cowley LA, Perry NT, Adak G, et al. Whole-genome sequencing for national surveillance of Shiga toxin-producing Escherichia coli 0157. Clin Infect Dis. 2015;61(3):305-12. https://doi.org/10.1093/cid/civ318 PMID: 25888672

7. Byrne L, Adams N, Glen K, Dallman TJ, Kar-Purkayastha I, Beasley G, et al. Epidemiological and Microbiological Investigation of an Outbreak of Severe Disease from Shiga Toxin-Producing Escherichia coli O157 Infection Associated with Consumption of a Slaw Garnish. J Food Prot. 2016;79(7):1161-8. https://doi.org/10.4315/0362-028X.JFP-15 580 PMID: 27357035

8. International Organisation for Standards. Protocols for the International Standard methods. Geneva: ISO; 2013 Available from: https://www.iso.org/home.html

9. Jenkins C, Dallman TJ, Launders N, Willis C, Byrne L, Jorgensen F, et al. Public Health Investigation of Two Outbreaks of Shiga Toxin-Producing Escherichia coli 0157 Associated with Consumption of Watercress. Appl Environ Microbiol. 2015;81(12):3946-52. https://doi.org/10.1128/AEM.04188-14 PMID: 25841005

10. Pritchard GC, Smith R, Ellis-Iversen J, Cheasty T, Willshaw GA Verocytotoxigenic Escherichia coli $0_{157}$ in animals on public amenity premises in England and Wales, 1997 to 2007. Vet Rec. 2009;164(18):545-9. https://doi.org/10.1136/vr.164.18.545 PMID: 19411683

11. Jenkins C, Lawson AJ, Cheasty T, Willshaw GA. Assessment of a real-time PCR for the detection and characterization of verocytotoxigenic Escherichia coli. J Med Microbiol. 2012;61):1082-5. https://doi.org/10.1099/jmm.0.041517-0 PMID: 22516135

12. Li H, Durbin R. Fast and accurate long-read alignment with Burrows-Wheeler transform. Bioinformatics. 2010;26(5):58995. https://doi.org/10.1093/bioinformatics/btp698 PMID: 20080505

13. McKenna A, Hanna M, Banks E, Sivachenko A, Cibulskis K, Kernytsky A, et al. The Genome Analysis Toolkit: a MapReduce framework for analyzing next-generation DNA sequencing data. Genome Res. 2010;20(9):1297-303. https://doi.org/10.1101/ gr.107524.110 PMID: 20644199

14. Stamatakis A. RAxML version 8: a tool for phylogenetic analysis and post-analysis of large phylogenies.

Bioinformatics. 2014;30(9):1312-3. https://doi.org/10.1093/ bioinformatics/btu033 PMID: 24451623

15. Ashton PM, Perry N, Ellis R, Petrovska L, Wain J, Grant KA, et al. Insight into Shiga toxin genes encoded by Escherichia coli 0157 from whole genome sequencing. PeerJ. 2015;3:e739. https://doi.org/10.7717/peerj.739 PMID: 25737808

16. Wilson D, Dolan G, Aird H, Sorrell S, Dallman TJ, Jenkins C, et al. Farm-to-fork investigation of an outbreak of Shiga toxinproducing Escherichia coli 0157. Microb Genom. 2018;4(3). https://doi.org/10.1099/mgen.0.000160 PMID: 29488865

17. Willis C, Jørgensen F, Aird H, Elviss N, Fox A, Jenkins C, et al. An assessment of the microbiological quality and safety of raw drinking milk on retail sale in England. J Appl Microbiol. 2018;124(2):535-46. https://doi.org/10.1111/jam.13660 PMID: 29215775

18. McLauchlin J, Aird H, Charlett A, Chattaway M, Elviss N, Hartman $\mathrm{H}$, et al. Imported edible leaves collected at retail sale in England during 2017 with an emphasis on betel and curry leaves: microbiological quality with respect to Salmonella, Shiga-toxin-producing E. coli (STEC) and levels of Escherichia coli. J Appl Microbiol. 2018;125(4):1175-85.

19. Butcher H, Elson R, Chattaway MA, Featherstone CA, Willis $C$, Jorgensen $\mathrm{F}$, et al. Whole genome sequencing improved case ascertainment in an outbreak of Shiga toxin-producing Escherichia coli 0157 associated with raw drinking milk. Epidemiol Infect. 2016;144(13):2812-23. https://doi. org/10.1017/S0950268816000509 PMID: 27338677

20. Ethelberg S, Olsen KE, Scheutz F, Jensen C, Schiellerup $P$, Enberg J, et al. Virulence factors for hemolytic uremic syndrome, Denmark. Emerg Infect Dis. 2004;10(5):842-7. https://doi.org/10.3201/eid1005.030576 PMID: 15200817

21. Food Standards Agency (FSA). Update on Food Standards Agency review of controls for raw drinking milk (RDM) in England, Wales and Northern Ireland and proposals for future controls. London: FSA; 2014. Available from: http://www.food. gov.uk/sites/default/files/multimedia/pdfs/board/boardpapers2014/fsa-140704.pdf

22. The National Archives, Her Majesty's Stationery Office (HMSO). The Food Hygiene (England) Regulations 2006. Norwich: HMSO; 2006. Available from: http://www.legislation.gov.uk/ uksi/2006/14/pdfs/uksi_20060014_en.pdf

23. Food Standards Agency (FSA). Raw Drinking Milk Programme (RDM). London: FSA; 2018. Available from: https://www.food. gov.uk/sites/default/files/fsa180307.pdf

24. Smith-Palmer A. Incident Management Team Report.

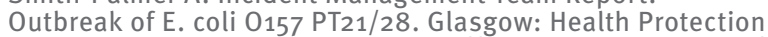
Scotland; 2017. Available from: http://www.hps.scot.nhs.uk/ resourcedocument.aspx? $\mathrm{id}=5844$

25. European Food Safety Authority (EFSA). Scientific Opinion on the public health risks related to the consumption of raw drinking milk. EFSA J. 2015;13(1):3940.

26. Strachan NJC, Hunter CJ, Jones CDR, Wilson RS, Ethelberg $\mathrm{S}$, Cross $\mathrm{P}$, et al. The relationship between lay and technical views of Escherichia coli 0157 risk. Philos Trans R Soc Lond B Biol Sci. 2011;366(1573):1999-2009. https://doi.org/10.1098/ rstb.2010.0393 PMID: 21624920

\section{License, supplementary material and copyright}

This is an open-access article distributed under the terms of the Creative Commons Attribution (CC BY 4.0) Licence. You may share and adapt the material, but must give appropriate credit to the source, provide a link to the licence and indicate if changes were made.

Any supplementary material referenced in the article can be found in the online version.

This article is copyright of the authors or their affiliated institutions, 2019. 